\title{
Auf den Punkt gebracht
}

\author{
To the Point
}

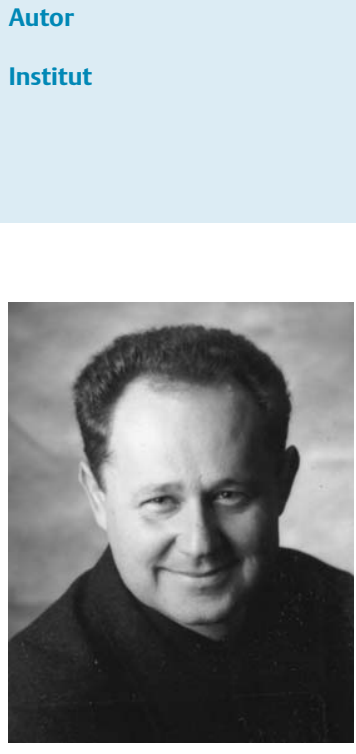

Prof. Dr. Manfred Wildner

Bibliografie

DOI $10.1055 / \mathrm{s}-0028-1085436$ Gesundheitswesen 2008;

70: 517-518

(c) Georg Thieme Verlag KG Stuttgart · New York

ISSN 0941-3790

\section{Korrespondenzadresse}

\section{Prof. Dr. med. M. Wildner}

Bayerisches Landesamt für Gesundheit und Lebensmittelsicherheit

Veterinärstraße 2

85762 Oberschleissheim

manfred.wildner@Igl.bayern.de

\section{Wildner}

Bayerisches Landesamt für Gesundheit und Lebensmittelsicherheit, Oberschleissheim + Foto von Herrn Wildner aus Heft 6/08 übernehemen
„Die Menschheit hat in Zauber und Gebet das Schicksal zu wenden gesucht, sie hat mit Mitteln, denen keine Wirkung zukommt, Krankheiten bekämpft und auf viele andere Weise ihre Kräfte unnütz und schädlich angewendet [...]. All das ist das Resultat eines Denkens, das keine Rücksicht nimmt auf die Grenzen der Erfahrung, und das auf eine Kontrolle der Resultate an der Wirklichkeit und eine logische Kritik verzichtet, d.h. analog und in gewissem Sinne geradezu identisch ist mit dem Denken im Traume und dem des autistischen Schizophrenen [...]. Es ist deshalb das autistische Denken genannt worden. Dieses hat seine besonderen, von der (realistischen) Logik abweichenden Gesetze, es sucht nicht Wahrheit, sondern Erfüllung von Wünschen“ ([1], S. 1).

Als der Züricher Psychiater Eugen Bleuler 1919 seinen Aufsatz „Das autistisch-undisziplinierte Denken in der Medizin und seine Überwindung“ veröffentlichte, aus dem dieses Zitat stammt, waren randomisierte kontrollierte Studien und Placebo-Behandlungen als Ausweg noch nicht erdacht worden. Dennoch hatte Bleuler eine Idee, welche in diese Richtung zielte: die „Udenotherapie“, wie er das abwartende, vorurteilsfreie Zusehen frei von blindem Aktionismus nannte.

Ein bloß historisch mehr oder weniger interessantes Zitat? Ein spätes Bekenntnis zum etablierten Programm der Aufklärung, von Immanuel Kant bereits 1784 in der Berlinischen Monatsschrift als „Ausgang des Menschen aus seiner selbstverschuldeten Unmündigkeit" auf den Punkt gebracht? Wohl nicht - Eugen Bleulers Mahnung ist unverändert aktuell, auch nach der Etablierung randomisierter kontrollierter Studiendesigns (RCT) in Zusammenhang mit der Chemotherapie der Tuberkulose 1948 [2] und von CochraneZentren weltweit und auch in Deutschland (www.cochrane.de). Im Kontext der evidenzbasierten Medizin wird RCTs als „Königsweg“ der Therapiestudien besonderes Gewicht bei entsprechend gelagerten Forschungsfragen zuer- kannt. Auf die Diskussion dazu im deutschen Sprachraum sei hingewiesen $[3,4]$.

Dass dieses Design auch für Fragen der Wirksamkeit alternativer Heilverfahren angewendet werden kann, steht in Expertenkreisen außer Frage. Um Eugen Bleuler noch einmal zu zitieren: „Ich habe einen Freund, der Homöopath ist und mit den Spitzen seiner Gesinnungsgenossen in engem Kontakt steht. Er ist [...] von seinen Theorien in gleicher Weise überzeugt, wie jeder von uns von den seinigen, und er kann gute praktische Resultate zu seinen Gunsten anführen. Aber mich kann er nicht überzeugen, weil er keinen direkten Vergleich bringen kann, nicht nur mit der Allopathie, sondern, was viel wichtiger, mit der Udenotherapie, wenn es erlaubt ist, den Ausdruck zu brauchen." [1, S. 16f].

Der Ausdruck mag erlaubt sein, das Vorgehen der reinen Beobachtung („Udenotherapie“) eines Vergleichsarmes nur mehr bedingt. Ethische Prinzipien erfordern seit der zweiten Hälfte des 20.Jahrhunderts die sorgfältige Abwägung der Therapie innerhalb des Kontrollarmes bzw. der Kontrollarme. Zur Auswahl stehen oft die übliche Versorgungsroutine („standard of care“) und die Scheinbehandlungen („Placebo“).

Ein schönes Beispiel eines hochwertigen mehrarmigen Designs einer Therapiestudie zu einem Alternativverfahren sind die Akupunkturstudien der Gesetzlichen Krankenversicherungen $[5,6]$. Die Ergebnisse sind spannend: Sowohl klassische Akupunktur als auch Scheinakupunktur sind der medikamentösen Standardtherapie bei verschiedenen Indikationen überlegen, wobei sich klassische Akupunktur und Scheinakupunktur bis auf eine Indikation in ihrer Wirksamkeit untereinander nicht unterscheiden.

Ein Beweis für die Wirksamkeit der Akupunktur? Um die Ergebnisse auf den Punkt zu bringen: Akupunktur und Scheinakupunktur scheinen in den beiden Verfahren gemeinsamen Anteilen eine Wirkung zu entfalten, welche derjenigen der me- 
dikamentengestützten Therapie überlegen ist. In dem spezifischen Anteil der Nadelung klassischer Akupunkturpunkte scheint demgegenüber keine zusätzliche Wirksamkeit zu liegen. Die Wirksamkeit wäre demnach den Anteilen der intensivierten und spezifischen Arzt-Patienten-Interaktion zuzuordnen sowie möglicherweise dem Ritual der Nadelung - gleich wo die Nadelspitze zu liegen kommt.

Ein einfacher Placebo-Effekt also? Das Adjektiv „einfach“ ist bei Placebo-Effekten fehl am Platz: Diese scheinen komplex vermittelt zu werden, wenn sie gegenüber der Option „keine Therapie“ (Bleulers „Udenotherapie“) überhaupt eine erhöhte Wirksamkeit haben [7].

Was Placebos wirksam macht? Zum Beispiel Erwartungen, Qualität der Interaktion, Rituale: Diese drei Faktoren entfalten offenbar Heilkräfte, die sich summieren lassen und in ihrer Wirksamkeit Medikamenten u.U. gleichwertig oder überlegen sind [8]. Dies „einfach“ als Placebo-Effekt abzutun, ist irrig. Placebo ist eben nicht gleich Placebo, „der Kontext zählt“. Zugespitzt könnten die Studienergebnisse der o.g. Akupunkturstudie zukunfts- und erkenntnisorientiert als ein starkes Plädoyer für eine spezifische Placeboforschung oder auch für eine Forschung zu einer „sprechenden Medizin“ interpretiert werden.

Warum diese auf den ersten Blick vielleicht spitzfindig wirkende Unterscheidung? Werden hier nicht einfache Sachverhalte unnötig verkompliziert, womöglich aus einem voreingenommenen „schulmedizinischen“ Denken heraus? Ist die genauere Bestimmung der wirksamen Komponenten überhaupt notwendig? Gilt nicht die einfache Aussage „Akupunktur ist wirksam, insbesondere wirksamer als eine Standardtherapie mit Medikamenten“? Und: „wer heilt, hat Recht“?

Richtig ist, dass die Dinge nicht komplizierter gemacht werden sollten, als sie sind - jedoch auch nicht einfacher. Gegen ein verfälschend-simplifizierendes Verständnis des Sachverhaltes wird vorsorglich Widerspruch eingelegt. Eine rhetorisch reizvolle Begründung für diesen Widerspruch findet sich in dem Essay von Harry G. Frankfurt: Bullshit [9]. Der prägnante Titel kann auf Vorschlag des Autors synonym und für die weitere Konversation etwas gefälliger auch mit Humbug bzw. Bluff wiedergegeben werden [ebd., S. 12]. Als zentrales Kennzeichen von Humbug bzw. Bluff wird eine fehlende Ernsthaftigkeit in der Suche nach Wahrheit festgestellt: gerade „in dieser Gleichgültigkeit gegenüber der Frage, wie die Dinge wirklich sind [...] liegt meines Erachtens das Wesen des Bullshit“ [ebd., S. 40]; „anders als der aufrichtige Mensch und als der Lügner achtet er [der dem Humbug verfallene Mensch] auf die Tatsachen nur in so weit, als sie für seinen Wunsch, mit seinen Behauptungen durchzukommen, von Belang sein mögen“ [ebd. S. 63].

Die Parallelen des von Harry G. Frankfurt als „Bluff“ kritisierten Sprechens zu dem von Eugen Bleuler als „autistisch“ charakterisierten Denken sind offensichtlich (s. o.). Es ist bei genauerer Betrachtung eben nicht gleichgültig, ob das Wirkprinzip erfolgreicher Akupunktur in der Arzt-Patienten-Interaktion liegt oder ob es in der exakten Platzierung einer Nadelspitze entlang von Meridianen zu suchen ist. Ersteres ist eine allgemeinere Komponente therapeutischen Handelns, letzteres die spezifische Differenz, welche die Akupunktur recht eigentlich zur Akupunktur macht - wenn denn diese aus dem Lateinischen abgeleitete sprachliche Bezeichnung eine sinnvolle Bedeutung beansprucht.
Um noch einmal Eugen Bleulers Worte zu gebrauchen: „Irrtümer, nicht Lücken, hindern die Wissenschaft am Fortschreiten [...] Für die Engramme, die in unser Gehirn geschrieben werden, gibt es keinen Tintentod; einmal gewonnene Assoziationen lassen sich höchstens übertönen, niemals mehr auswischen“ [1, S.15-16]

Es ist nicht gleichgültig, ob die Praxis der Medizin sensibel gegenüber solchen feinen, dabei in vieler Hinsicht bedeutsamen Unterschieden ist oder ob indifferent. Es geht um den Erhalt eines exakten Denkens im Dienst am Menschen und an der Sache, um die Abwehr von Bequemlichkeit und Feigheit (Kant), von falsch verstandener Höflichkeit und Gefälligkeit (Bleuler), von Sich-Wichtig-Machen und fehlender Ernsthaftigkeit (Frankfurt). Harry G. Frankfurts drastischer Essay-Titel „Bullshit“ sei eine Mahnung am Beginn des 21. Jahrhunderts, so wie es Eugen Bleulers Aufsatztitel vor beinahe 100 Jahren war: Zu Ernsthaftigkeit und Aufrichtigkeit gegenüber den Sachverhalten und nicht nur gegenüber den eigenen Überzeugungen, zu Vorsicht gegenüber anti-realistischen Doktrinen und zu intellektueller, sprachlicher und handwerklicher Sorgfalt.

Um noch einen Punkt zu machen: Dies schließt die weitere ernsthafte Auseinandersetzung mit der Wirksamkeit alternativer Heilverfahren ausdrücklich ein. Alternativ bezogen auf eine zu Recht beklagte Engführung medizinischer Therapieoptionen hin zu chemischen Substanzen und operativen Eingriffen, nicht jedoch alternativ zu einer der Sache angemessenen, gedanklich stringenten wissenschaftlichen Wirksamkeitsforschung, Forschungsinterpretation und -rezeption.

Als Teil dieser Diskussion berichten Bücker et al. in diesem Heft von ihrer Studie mit telefonischen Befragungen von 1001 Personen zum Gebrauch alternativer Heilverfahren in der Allgemeinbevölkerung. Die Ergebnisse sind als „electronic long print short (ELPS)“-Beitrag festgehalten: In Kurzform sind sie in deutscher Sprache abgedruckt, die englischsprachige Langfassung findet sich im Internet (URL siehe dort).

\section{Literatur}

1 Bleuler E. Das autistisch-undisziplinierte Denken in der Medizin und seine Überwindung. Springer Berlin; 1919, hier: 4. Auflage 1927

2 Streptomycin treatment of pulmonary tuberculosis: a Medical Research Council investigation. BMJ 1948; 2: 769-782

3 Willich SN. Randomisierte kontrollierte Studien: Pragmatische Ansätze erforderlich. In: Deutsches Ärzteblatt Jg. 103, Nr. 39, 29.09.2006. Köln: Deutscher Ärzte-Verlag; S A2524-S A2529

4 Windeler J, Antes G, Behrens J et al. Randomisierte kontrollierte Studien: Kritische Evaluation ist ein Wesensmerkmal ärztlichen Handelns. In: Deutsches Ärzteblatt Jg. 105, Nr. 11, 14.03.2008. Deutscher Ärzte-Verlag Köln, SA-565

5 Witt CM, Brinkhaus B, Willich SN. Akupunktur. Klinische Studien zur Wirksamkeit bei Patienten mit chronischen Schmerzen. Bundesgesundheitsblatt Gesundheitsforschung Gesundheitsschutz 2006; 49 (8): 736-742

6 Witt CM, Jena S, Selim D et al. Pragmatic randomized trial evaluating the clinical and economic effectiveness of acupuncture for chronic low back pain. Am J Epidemiol 2006; 164 (5): 487-496

7 Hróbjartsson A, Gøtzsche PC. Is the placebo powerless? Update of a systematic review with 52 new randomized trials comparing placebo with no treatment. J Intern Med 2004; 256 (2): 91-100

8 Kaptchuk TJ, Kelley JM, Conboy LA et al. Components of placebo effect: randomised controlled trial in patients with irritable bowel syndrome. BMJ 2008; 336 (7651): 999-1003

9 Frankfurt HG. Bullshit. Suhrkamp Verlag Frankfurt a.M. 2006, [Originalausgabe: On Bullshit. Princeton University Press, 2005] 